\title{
Preface
}

\section{PET in the Era of Theranostics}

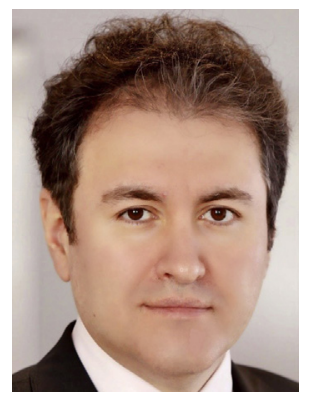

Hojjat Ahmadzadehfar, MD, MSc

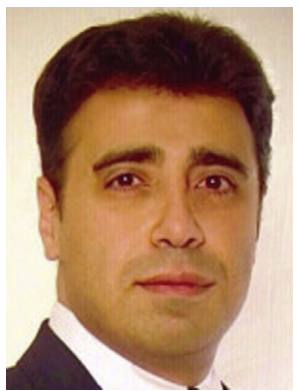

Ali Gholamrezanezhad, MD, FEBNM, DABR

Editors
The heterogeneity of human malignancies has led the oncology community to the integration of modern imaging modalities into their management and the most critical components of treatment planning in this population. By providing accurate staging, molecular characterization, and accurate quantification of disease burden, clinicians are guided for optimal management of numerous cancers.

Combined diagnosis and therapy by radioactive agents originated with the groundbreaking initiative by Saul Hertz (1905-1950), who performed the first radioiodine therapy in 1941. This innovative approach started a new beginning in medical treatment and, over the last 2 decades, resulted in our growing knowledge about molecular oncology and tumor immunology. These developments together have generated the concept of theranostics, which bridges the gap between therapeutic interventions and diagnostics. In the field of molecular medicine, theranostics combines imaging and therapy techniques that use the same or similar molecules, either radiolabeled differently or administered with different dosages. This strategy makes it possible to select eligible patients for a specific therapy and, in turn, enhances response rate, and therefore, overall survival while minimizing side effects.

Adopting molecular imaging modalities, particularly PET, in treatment planning has shown significant benefits with regard to the outcomes, including progression-free and overall survival of patients with cancer. Furthermore, this approach has led to substantial improvement of clinical symptoms of the affected population. Most notably, personalized radionuclide therapy in the framework of theranostics provides promising outcomes and treatment responses with significantly decreased adverse effects, such as hematologic or nephrologic toxicity, resulting in improved quality of life for patients with various cancers.

The field of personalized medicine is rapidly adopting PET, single-photon emission computed tomography, optical imaging, and to some extent, MRI (including magnetic resonance [MR] fingerprinting and MR spectroscopy) to identify and visualize potential molecular targets and unmask the molecular phenotype of the neoplastic cells. 
Numerous novel radiotracers are used as theranostic probes in clinical practice, among which ${ }^{68} \mathrm{Ga}$-labeled somatostatin receptor agents, ${ }^{177} \mathrm{Lu}$-based radiopharmaceuticals, and radiolabeled antibodies are especially noteworthy. This issue of PET Clinics aims to provide a summary of contextual knowledge and current clinical applications of theranostics in modern oncology. The comprehensive review articles of the subject selected for this issue of PET Clinics allow scientists, oncologists, and other specialists to realize the great potential of this innovative approach and encourage future studies in this expanding area of molecular imaging in medicine.

In this issue, Jose Flavio Marin and his colleagues outline the general concepts and basics of theranostics. Other articles cover the role of theranostics in the management of various cancers, including prostate, neuroendocrine, brain, and thyroid cancers and neuroblastoma. Noncancer-related applications of theranostics are also discussed and include management of musculoskeletal diseases. We hope the articles in this issue compellingly illustrate what is known about theranostics, highlight gaps in current knowledge, and help point to promising opportunities for future research in this very exciting discipline in medicine.

Hojjat Ahmadzadehfar, MD, MSc

Department of Nuclear Medicine

Klinikum Westfalen

Am Knappschaftskrankenhaus 1

Dortmund 44301, Germany

Ali Gholamrezanezhad, MD, FEBNM, DABR

Department of Radiology-LAC+USC

1983 Marengo Street

Los Angeles, CA 90033, USA

E-mail addresses:

hojjat.ahmadzadehfar@ruhr-uni-bochum.de

(H. Ahmadzadehfar)

gholamre@usc.edu (A. Gholamrezanezhad) 